\title{
PRESSURE DISTRIBUTION IN A SQUEEZE FILM SPHERICAL BEARING WITH ROUGH SURFACES LUBRICATED BY AN ELLIS FLUID
}

\author{
P. JURCZAK* and J. FALICKI \\ University of Zielona Góra, Faculty of Mechanical Engineering \\ ul. Szafrana 2, P.O.Box 47, 65-516 Zielona Góra, POLAND \\ E-mails: P.Jurczak@ibem.uz.zgora.pl \\ J.Falicki@ibem.uz.zgora.pl
}

\begin{abstract}
In this paper, the solution to a problem of pressure distribution in a curvilinear squeeze film spherical bearing is considered. The equations of motion of an Ellis pseudo-plastic fluid are presented. Using Christensen's stochastic model of rough surfaces, different forms of Reynolds equation for various types of surface roughness pattern are obtained. The analytical solutions of these equations for the cases of externally pressurized bearing and squeeze film bearing are presented. Analytical solutions for the film pressure are found for the longitudinal and circumferential roughness patterns. As a result the formulae expressing pressure distribution in the clearance of bearing lubricated by an Ellis fluid was obtained. The numerical considerations for a spherical bearing are given in detail.
\end{abstract}

Key words: pseudo-plastic model of Ellis fluid, thrust curvilinear bearings, modified Reynolds equation

\section{Introduction}

The phenomenon of the squeeze film of a non-Newtonian fluid may be found in many applications such as: bioengineering, stamping, molding, lubrication technology, metal processing, pressure die casting, polymer processing [1-3].

In recent years the problem of the influence of the bearing surface roughness on the mechanical parameters of the bearing has received considerable attention, because the bearing surfaces are all rough. The roughness asperities are, generally speaking, of the same order as the bearing thickness. Under these conditions surface roughness has a considerable influence on the mechanical parameters of a bearing operating even in the hydrodynamic lubrication.

With the progress of modern machine elements the growing use of composite fluids as lubricants has become of large interest. The rheological properties of composite lubricants have been modelled by nonNewtonian fluids, for example: the micropolar [3], couple-stress [4], viscoplastic [5,6], pseudo-plastic [7-10] and mixture [11]. A more complete list of the non-Newtonian models may be found in [12-14].

The flows of Newtonian and non-Newtonian fluids in the clearance with impermeable and smooth walls have been examined theoretically and experimentally. The clearance walls have been modelled as two disks, two conical or spherical surfaces [15-18]. A more general case is established by a flow in the clearance created by two surfaces of revolution [19].

The basis of this paper is the stochastic theory of hydrodynamic lubrication of rough surfaces developed by Christensen [20]. This model has been applied to the study of the surface roughness of different geometrical configurations in many papers [21-29].

Based on the Christensen stochastic theory Gururajan and Prakash [30] have been obtained the modified Reynolds equation applicable to two types of directional roughness structure. Walicka and coworkers [26-29] used this equation to designate mechanical parameters of the rough bearing with squeeze film.

\footnotetext{
* To whom correspondence should be addressed
} 
The aim of this paper is a general study of the pressure distribution in two types of bearings, namely:

- externally pressurized bearings,

- squeeze film bearings.

The pseudo-plastic lubricant behaviour is described by the model of the Ellis fluid [31] and the Christensen stochastic theory of hydrodynamic lubrication of rough surfaces is used [20]. The different forms of Reynolds equation for various cases of surface roughness pattern are obtained. Analytical solutions for the squeeze film pressure for various types of surface roughness are presented.

\section{Derivation of the modified Reynolds equation}

The lubricating oils and some greases can be described by the model of a non-Newtonian fluid. Many lubricants have the same characteristics as pseudo-plastic fluids. Rotem and Shinnar [32] presented a method for expressing experimentally the relation linking the stress and the shear rate as

$$
\frac{d \gamma}{d t}=\frac{\tau}{\mu}\left(1+\sum_{i=-1}^{n} k_{i} \tau^{2 i}\right)
$$

Keeping only the first and the last order terms the relation (2.1) we obtain the Ellis model [31]

$$
\mu \frac{d \gamma}{d t}=\tau+k_{n} \tau^{n}
$$

In this paper, the original notation given by Ellis [31] will be used:

$$
\frac{d \gamma}{d t}=\frac{\tau}{\mu_{N}}=\frac{\tau}{\mu}\left(1+\left|\frac{\tau}{\tau_{1 / 2}}\right|^{n-1}\right)
$$

where $\tau_{l / 2}$ is the characteristic shear stress at which $\mu=2 \mu_{N}\left(\mu_{N}\right.$ - Newtonian viscosity), $n$ is the model indicial parameter.

Taking into account the considerations of the works $[10,34]$ the three-dimensional notation of Eq.(2.3) may be presented as follows

$$
\mu \boldsymbol{A}_{1}=\Lambda\left\{1+\frac{1}{\tau_{l / 2}^{n-1}} \Lambda^{n-1}\right\}, \quad \Lambda=\left[\frac{1}{2} \operatorname{tr}\left(\Lambda^{2}\right)\right]^{\frac{1}{2}},
$$

where: $\Lambda$ is the magnitude of the second-order shear stress tensor $\boldsymbol{\Lambda}$, but $\boldsymbol{A}_{l}$ is the first Rivlin-Ericksen kinematic tensor.

Let us consider a thrust curvilinear bearing shown in Fig.1. Both the working surfaces of this bearing are rough as shown in Fig.1. The lower surface of the bearing is described by the function $R(x)$ which is the radius of this surface. The nominal bearing clearance thickness is described by the expression $H(x, t)$ which is equal to the sum

$$
H=h(x, t)+h_{S}(x, \vartheta, \xi)
$$


where $h(x, t)$ is the nominal smooth part of the film geometry, while $h_{s}=\delta_{r}+\delta_{s}$ denotes the random part resulting from the surface roughness asperities measured from the nominal level, $\xi$ describes a random variable which characterizes the definite roughness arrangement. An intrinsic curvilinear orthogonal coordinate system $x, \vartheta, y$ linked with the lower bearing surface is also presented in Fig.1.
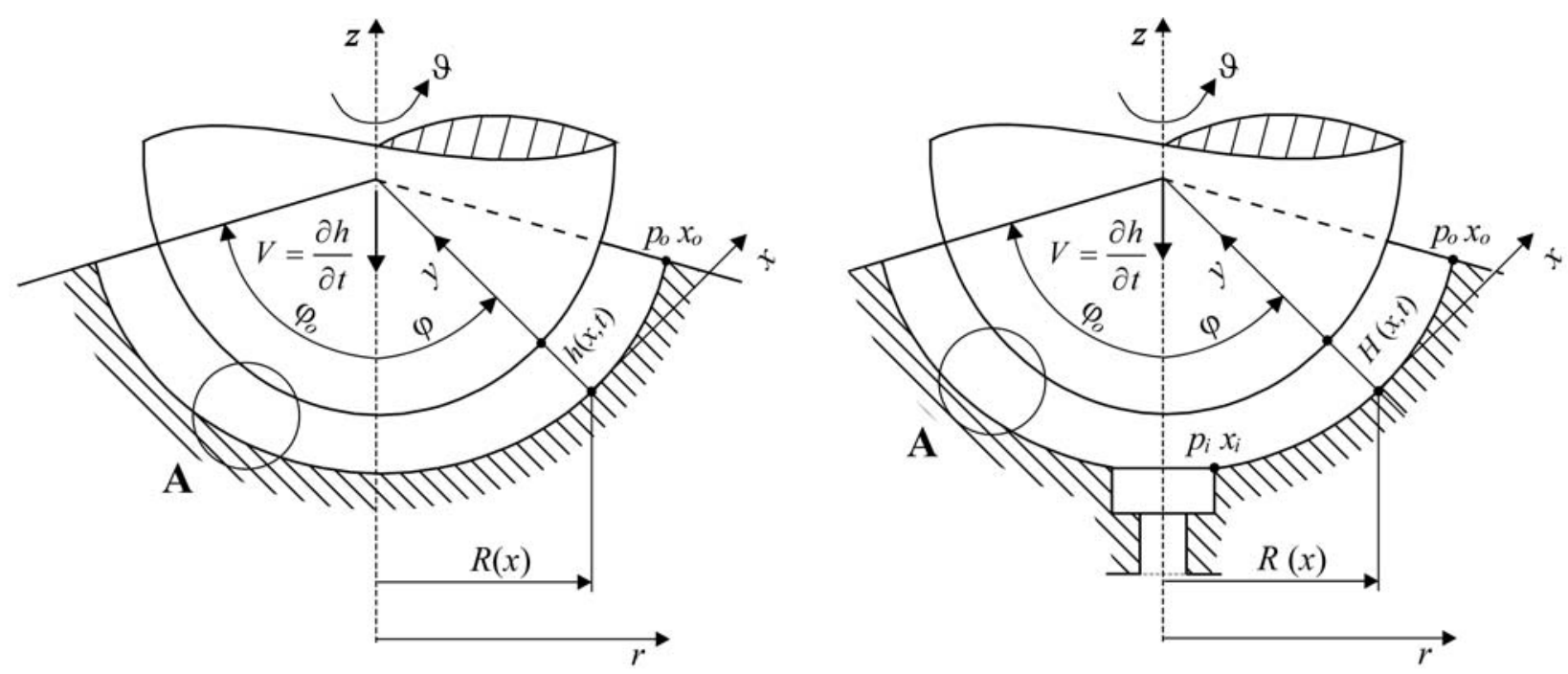

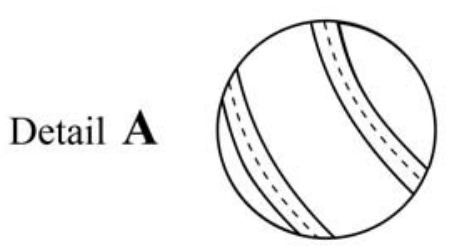

Longitudinal roughness (radial)

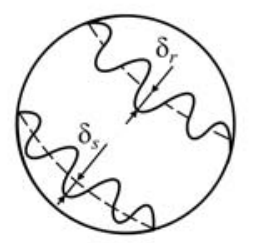

Circumferential roughness (transverse)

Fig.1. Configuration of a curvilinear thrust bearing with rough surfaces.

Using the assumptions typical for the flows in a thin layer $[10,11,34]$ the equations of motion for an Ellis fluid for axial symmetry can be presented in the following form

$$
\begin{aligned}
& \frac{1}{R} \frac{\partial\left(R v_{x}\right)}{\partial x}+\frac{\partial v_{y}}{\partial y}=0, \\
& \frac{\partial \Lambda_{x y}}{\partial y}=\frac{\partial p}{\partial x}, \quad \frac{\partial p}{\partial y}=0, \\
& \mu \frac{\partial v_{x}}{\partial y}=\Lambda_{x y}\left[1+\left(\frac{\left|\Lambda_{x y}\right|}{\tau_{1 / 2}}\right)^{n-1}\right]
\end{aligned}
$$

The boundary conditions for the velocity components are stated as follows

$$
\mathrm{v}_{x}(x, 0, t)=0, \quad \mathrm{v}_{x}(x, H, t)=0,
$$




$$
v_{y}(x, 0, t)=0, \quad v_{y}(x, h, t)=\frac{\partial H}{\partial t}=\dot{H},
$$

Solving the system of equations of motion (2.6), (2.7) and taking into account the constitutive equation (2.8) one obtains the following form of the modified Reynolds equation

$$
\frac{1}{R} \frac{\partial}{\partial x} R H^{3}\left(-\frac{\partial p}{\partial x}\right)\left[1+\frac{3}{n+2}\left(\frac{H}{2 \tau_{1 / 2}}\right)^{n-1}\left(-\frac{\partial p}{\partial x}\right)^{n-1}\right]=-12 \mu \frac{\partial h}{\partial t}
$$

for a pseudo-plastic lubricant of Ellis type. If $n=1$, and taking into account that $\mu=2 \mu_{N}$, Eq.(2.11) reduces to the Reynolds equation for a Newtonian fluid [36].

If the film thickness is regarded as a random quantity, a height distribution function must be associated. Many real bearing surfaces show a roughness height distribution which is closely Gaussian, at least up to three standard deviations. From a practical point of view, the Gaussian distribution is rather inconvenient and therefore a polynomial form of its approximation is chosen. Following Christensen [25, 35, 36] such a probability density function is given by

$$
f\left(h_{s}\right)= \begin{cases}\frac{35}{32 c^{7}}\left(c^{2}-h_{s}^{2}\right)^{3}, & -c \leq h_{s} \leq+c \\ 0, & \text { elsewhere }\end{cases}
$$

where $c$ is the half total range of the random film thickness variable. The function terminates at $c= \pm 3 \sigma$, where $\sigma$ is the standard deviation. equation

Inserting expected values in Eq.(2.11) we obtain the general form of the stochastic Reynolds

$$
\frac{1}{R} \frac{\partial}{\partial x}\left(E\left\{R H^{3}\left(-\frac{\partial p}{\partial x}\right)\left[1+\frac{3}{n+2}\left(\frac{H}{2 \tau_{1 / 2}}\right)^{n-1}\left(-\frac{\partial p}{\partial x}\right)^{n-1}\right]\right\}\right)=-12 \mu \frac{\partial E(H)}{\partial t}
$$

or

$$
\frac{1}{R} \frac{\partial}{\partial x} R\left(E\left(H^{3}\right)\left[-\frac{\partial(E p)}{\partial x}\right]\left\{1+\frac{3 E\left(H^{n-1}\right)}{(n+2)\left(2 \tau_{1 / 2}\right)^{n-1}}\left[-\frac{\partial(E p)}{\partial x}\right]^{n-1}\right\}\right)=-12 \mu \frac{\partial E(H)}{\partial t}
$$

where $E(\bullet)$ is the expectancy operator defined by

$$
E(\bullet)=\int_{-c}^{+c}(\bullet) f\left(h_{s}\right) d h_{s}
$$

The values of exponent $n$ for the Ellis fluid is included in the interval [37]

$$
0.25 \leq n \leq 4.0
$$


The problem is now reduced to devising means of evaluating the left-hand side of Eq.(2.14) subject to the specific model of roughness. The calculation of the mean film pressure distribution would require the evaluation of the expected value of various film thickness functions. For the probability density function given by Eq.(2.12) we have - for an integer exponents - the following expected values of film thickness functions $[26,27]$

$$
\begin{aligned}
& E(H)=h, \quad E\left(H^{2}\right)=h^{2}\left(1+\frac{1}{9} Y^{2}\right), \quad E\left(H^{3}\right)=h^{3}\left(1+\frac{1}{3} Y^{2}\right), \\
& E\left(H^{4}\right)=h^{4}\left(1+\frac{2}{3} Y^{2}+\frac{1}{33} Y^{4}\right), \quad E\left(H^{5}\right)=h^{5}\left(1+\frac{10}{9} Y^{2}+\frac{5}{33} Y^{4}\right), \\
& E\left(H^{6}\right)=h^{6}\left(1+\frac{5}{93} Y^{2}+\frac{5}{11} Y^{4}+\frac{5}{429} Y^{6}\right) \\
& E\left(H^{-1}\right)=\frac{1}{h}\left\{\frac{35}{32} \frac{1}{Y^{7}}\left[\left(Y^{2}-1\right) \ln \frac{1+Y}{1-Y}-\frac{2}{15} Y\left(15-40 Y^{2}+33 Y^{4}\right)\right]\right\}, \\
& E\left(H^{-2}\right)=\frac{1}{h^{2}}\left\{\frac{35}{32} \frac{1}{Y^{7}}\left[6\left(Y^{2}-1\right) \ln \frac{1+Y}{1-Y}-\frac{4}{5} Y\left(15-25 Y^{2}+8 Y^{4}\right)\right]\right\}, \\
& E\left(H^{-3}\right)=\frac{1}{h^{3}}\left\{\frac{35}{32} \frac{1}{Y^{7}}\left[3\left(5-Y^{2}\right)\left(Y^{2}-1\right) \ln \frac{1+Y}{1-Y}+2 Y\left(15-13 Y^{2}\right)\right]\right\}, \\
& E\left(H^{-4}\right)=\frac{1}{h^{4}}\left\{\frac{35}{32} \frac{1}{Y^{7}}\left[4\left(5-3 Y^{2}\right) \ln \frac{1+Y}{1-Y}-\frac{8}{3} Y\left(15-4 Y^{2}\right)\right]\right\}, \\
& E\left(H^{-5}\right)=\frac{1}{h^{5}}\left\{\frac{35}{32} \frac{1}{Y^{7}}\left[3\left(Y^{2}-5\right) \ln \frac{1+Y}{1-Y}+\frac{2 Y}{1-Y^{2}}\left(15-13 Y^{2}\right)\right]\right\}, \\
& E\left(H^{-6}\right)=\frac{1}{h^{6}}\left\{\frac{35}{32} \frac{1}{Y^{7}}\left[3 \ln \frac{1+Y}{1-Y}-\frac{2 Y}{5\left(1-Y^{2}\right)}\left(15-25 Y^{2}+8 Y^{4}\right)\right]\right\}
\end{aligned}
$$

where

$$
Y=\frac{c}{h}
$$

The expected values of film thickness functions $E\left(H^{-i}\right), i=1,2, \ldots, 6$, are not suitable for numerical purposes for small $Y$ values, therefore Taylor expansions in powers of $Y$ are proposed 


$$
\begin{aligned}
& E\left(H^{-1}\right)=\frac{1}{h}\left\{1+\sum_{n=1}^{\infty} \frac{105 Y^{2 n}}{(2 n+1)(2 n+3)(2 n+5)(2 n+7)}\right\}, \\
& E\left(H^{-2}\right)=\frac{1}{h^{2}}\left\{1+\sum_{n=1}^{\infty} \frac{105 Y^{2 n}}{(2 n+3)(2 n+5)(2 n+7)}\right\}, \\
& E\left(H^{-3}\right)=\frac{1}{h^{3}}\left\{1+\sum_{n=1}^{\infty} \frac{105(n+1) Y^{2 n}}{(2 n+3)(2 n+5)(2 n+7)}\right\}, \\
& E\left(H^{-4}\right)=\frac{1}{h^{4}}\left\{1+\sum_{n=1}^{\infty} \frac{35(n+1) Y^{2 n}}{(2 n+5)(2 n+7)}\right\}, \\
& E\left(H^{-5}\right)=\frac{1}{h^{5}}\left\{1+\sum_{n=1}^{\infty} \frac{35(n+1)(n+2) Y^{2 n}}{2(2 n+5)(2 n+7)}\right\}, \\
& E\left(H^{-6}\right)=\frac{1}{h^{6}}\left\{1+\sum_{n=1}^{\infty} \frac{7(n+1)(n+2) Y^{2 n}}{2(2 n+7)}\right\} .
\end{aligned}
$$

The first three formulae $(i=1,2,3)$ were given by Gururajan and Prakash [30], the next formulae are given by Walicka [26, 27].

For fractional exponents there are

$$
\begin{aligned}
& E\left(H^{m}\right)=h^{m}\left\{1+\sum_{n=1}^{\infty} \frac{105 Y^{2 n}}{2 n !(2 n+1)(2 n+3)(2 n+5)(2 n+7)} \prod_{i=1}^{2 n}(m-i+1)\right\}, \\
& E\left(H^{-m}\right)=\frac{1}{h^{m}}\left\{1+\sum_{n=1}^{\infty} \frac{105 Y^{2 n}}{2 n !(2 n+1)(2 n+3)(2 n+5)(2 n+7)} \prod_{i=1}^{2 n}(m+i-1)\right\} .
\end{aligned}
$$

\section{Solutions to the stochastic Reynolds equation}

Let us consider the Ellis fluid for which there is

$$
\frac{3}{n+2}\left(\frac{H}{2 \tau_{1 / 2}}\right)^{n-1}\left(-\frac{\partial \bar{p}}{\partial x}\right)^{n-1}<<1
$$

taking into account Eq.(3.1) the solutions of the Reynolds equation (2.13) can be searched in a form of the sum $[9,38,39]$ 


$$
E p=E p^{(0)}+E p^{(1)}
$$

Assuming that $E p^{(1)}<<E p^{(0)}$ and putting Eq.(3.2) into Eq.(2.13) we obtain two linearized equations

$$
\frac{1}{R} \frac{\partial}{\partial x} R\left\{E\left(H^{3}\right)\left[-\frac{\partial\left(E p^{(0)}\right)}{\partial x}\right]\right\}=-12 \mu \frac{\partial E(H)}{\partial t}
$$

and

$$
\frac{1}{R} \frac{\partial}{\partial x} R\left\{E\left(H^{3}\right)\left[-\frac{\partial\left(E p^{(1)}\right)}{\partial x}\right]\right\}=-\frac{3}{n+2} \frac{1}{R} \frac{\partial}{\partial x} R\left\{\frac{E\left(H^{n+2}\right)}{\left(2 \tau_{1 / 2}\right)^{n-1}}\left[-\frac{\partial\left(E p^{(0)}\right)}{\partial x}\right]\right\}
$$

In the present study, two types of roughness are of interest: the longitudinal (radial) one-dimensional roughness pattern, having the form of long narrow ridges and valleys running in the $x$ direction, and the circumferential (transverse) one-dimensional roughness pattern, having the form of long narrow ridges and valleys running in the $\vartheta$ direction $[26,28,29]$.

For the longitudinal one-dimensional roughness the film thickness is given by the expression

$$
H=h(x, t)+h_{s}(\vartheta, \xi)
$$

and the stochastic Reynolds equation (3.3) and (3.4) remain without change.

For the circumferential one-dimensional roughness the film thickness is given by the expression

$$
H=h(x, t)+h_{s}(x, \xi)
$$

and the stochastic Reynolds equations (3.3) and (3.5) take the form

$$
\frac{1}{R} \frac{\partial}{\partial x} R\left\{\frac{1}{E\left(H^{-3}\right)}\left[-\frac{\partial\left(E p^{(0)}\right)}{\partial x}\right]\right\}=-12 \mu \frac{\partial E(H)}{\partial t},
$$

and

$$
\begin{aligned}
& \frac{1}{R} \frac{\partial}{\partial x} R\left\{\frac{1}{E\left(H^{-3}\right)}\left[-\frac{\partial\left(E p^{(l)}\right)}{\partial x}\right]\right\}= \\
& =-\frac{3}{(n+2)\left(2 \tau_{1 / 2}\right)^{n-1}} \frac{1}{R} \frac{\partial}{\partial x} R\left\{\frac{1}{E\left(H^{-n-2}\right)}\left[-\frac{\partial\left(E p^{(0)}\right)}{\partial x}\right]^{n}\right.
\end{aligned}
$$

Note that we can present Eqs (3.3), (3.4) and Eqs (3.7), (3.8) in one common form 


$$
\begin{aligned}
& \frac{1}{R} \frac{\partial}{\partial x}\left[R H_{j}^{(3)}\left(-\frac{\partial \bar{p}^{(0)}}{\partial x}\right)\right]=-12 \mu \frac{\partial h}{\partial t}, \\
& \frac{1}{R} \frac{\partial}{\partial x}\left[R H_{j}^{(3)}\left(-\frac{\partial \bar{p}^{(1)}}{\partial x}\right)\right]=-\frac{3}{(n+2)\left(2 \tau_{1 / 2}\right)^{n-1}} \frac{1}{R} \frac{\partial}{\partial x}\left[R H_{j}^{(n+2)}\left(-\frac{\partial \bar{p}^{(0)}}{\partial x}\right)^{n}\right],
\end{aligned}
$$

where

$$
\bar{p}^{(0)}=E p^{(0)}, \quad \bar{p}^{(l)}=E p^{(l)}, \quad E(H)=h
$$

and

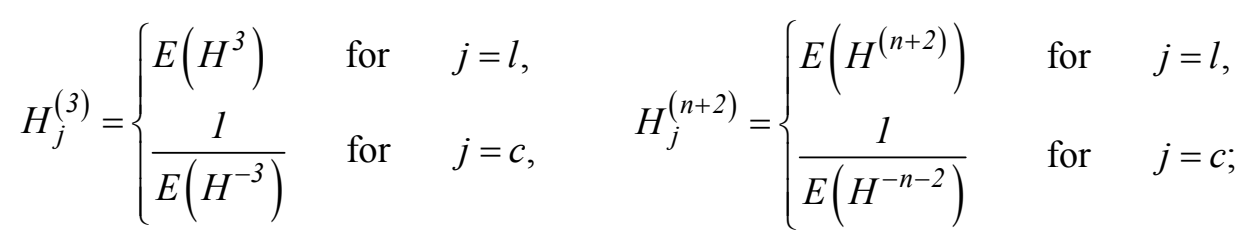

the case $j=l$ refers to the one-dimensional longitudinal roughness, but $j=c$ refers to the one-dimensional circumferential roughness.

The boundary conditions for pressure are:

- for the squeeze film bearing $\left(\frac{\partial h}{\partial t} \neq 0\right)$

$$
\left.\frac{\partial \bar{p}^{(0)}}{\partial x}\right|_{x=0}=0, \quad \bar{p}^{(0)}\left(x_{o}\right)=p_{o},\left.\quad \frac{\partial \bar{p}^{(l)}}{\partial x}\right|_{x=0}=\bar{p}^{(l)}\left(x_{o}\right)=0
$$

- for the externally pressurized bearing $\left(\frac{\partial h}{\partial t}=0\right)$

$$
\bar{p}^{(0)}\left(x_{i}\right)=p_{i}, \quad \bar{p}^{(0)}\left(x_{o}\right)=p_{o}, \quad \bar{p}^{(l)}\left(x_{i}\right)=\bar{p}^{(l)}\left(x_{o}\right)=0 .
$$

The solutions of Eqs (3.9) and (3.10) are given, respectively, as follows

$$
\bar{p}(x, t)=p_{o}-\left[F_{o}^{(n)}-F^{(n)}(x, t)\right]
$$

and

$$
\bar{p}=\alpha^{(n)} G^{(n)}(x)+\frac{\left[A(x)-A_{o}\right]\left(p_{i}-\alpha^{(n)} G_{i}^{(n)}\right)-\left[A(x)-A_{i}\right]\left(p_{o}-\alpha^{(n)} G_{o}^{(n)}\right)}{A_{i}-A_{o}}
$$


where

$$
\begin{aligned}
& I(x, t)=12 \mu \int \frac{\int R \dot{h} d x}{R H_{j}^{(3)}} d x, \\
& J^{(n)}(x, t)=\frac{3(12 \mu)^{n}}{(n+2)\left(2 \tau_{1 / 2}\right)^{n-1}} \int \frac{H_{j}^{(n+2)}}{R^{n}\left(H_{j}^{(3)}\right)^{n+1}}\left(-\int R \dot{h} d x\right)^{n} d x, \\
& F^{(n)}(x, t)=I(x, t)+J^{(n)}(x, t), \quad F_{o}=F\left(x_{o}, t\right) ; \\
& A(x)=\int \frac{d x}{R H_{j}^{(3)}}, \quad A_{o}=A\left(x_{o}\right), \\
& C=\frac{p_{i}-p_{o}}{A_{i}-A_{o}}, \quad \alpha^{(n)}=\frac{3}{n+2} \frac{(-C)^{n}}{\left(2 \tau_{1 / 2}\right)^{n-1}}, \\
& G^{(n)}(x)=\int \frac{H_{j}^{(n+2)} d x}{R^{n}\left(H_{j}^{(3)}\right)^{n+1}}, \quad G_{i}^{(n)}=G^{(n)}\left(x_{i}\right), \quad G_{o}^{(n)}=G^{(n)}\left(x_{o}\right) .
\end{aligned}
$$

\section{Spherical thrust squeeze film bearing}

Let us consider the spherical thrust squeeze film bearing shown in Fig.2.

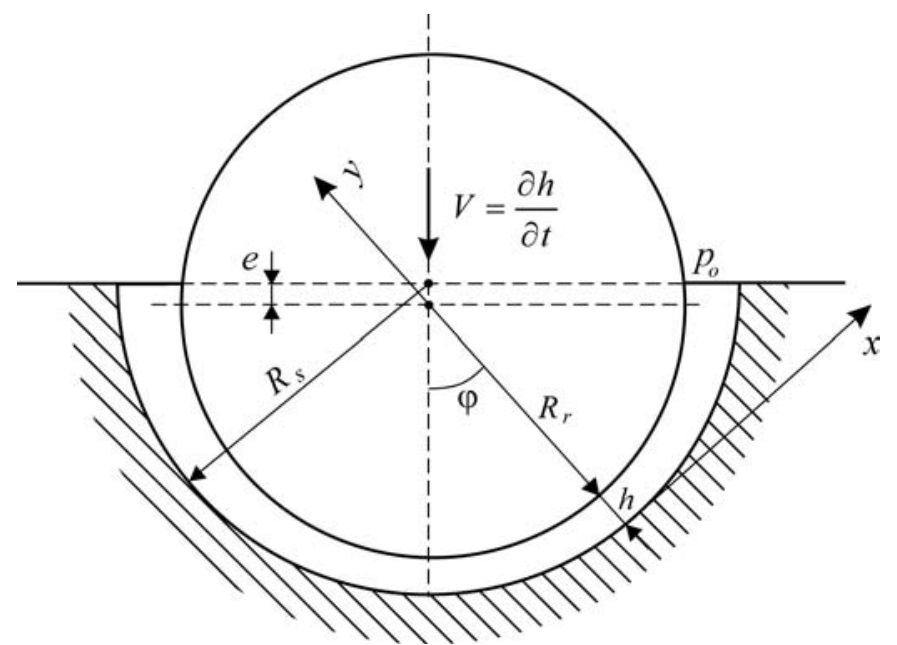

Fig.2. Spherical squeeze film bearing. 
Introducing the following parameters

$$
\begin{aligned}
& \tilde{x}=\frac{x}{R_{o}}, \quad x=R, \quad \tilde{R}=\frac{R}{R_{o}}, \quad C=R_{s}-R_{r}, \quad R=R_{r} \sin \varphi, \\
& \varphi=\frac{x}{R_{r}}, \quad \varepsilon(t)=\frac{e}{C}, \quad \dot{\varepsilon}=\frac{d \varepsilon}{d t}, \quad c^{*}=\frac{c}{C}, \\
& h=C(1-\varepsilon \cos \varphi), \quad \frac{\partial h}{\partial t}=\dot{h}=-C \dot{\varepsilon} \cos \varphi, \quad \tilde{h}=\frac{h}{C}=u=(1-\varepsilon \cos \varphi), \\
& \tilde{p}=\frac{\bar{p}(\varphi, t)-p_{o}}{\mu \dot{\varepsilon}}\left(\frac{C}{R_{r}}\right)^{2}, \quad \lambda^{(n)}=\left(\frac{\mu \dot{\varepsilon} x_{o}}{\tau_{1 / 2} h_{o}}\right)^{n-1} .
\end{aligned}
$$

we can present the following formula for the pressure distribution:

$$
\tilde{p}=6\left[\tilde{I}_{o}-\tilde{I}(\varphi, t)\right]-\frac{3(12)^{n}}{2^{2 n-1}(n+2)} \lambda^{n}\left[\tilde{J}_{o}^{(n)}-\tilde{J}^{(n)}(\varphi, t)\right]
$$

where

$$
\tilde{I}(\varphi, t)=\int \frac{\sin \varphi}{\tilde{H}_{j}^{(3)}} d \varphi \quad \text { and } \quad \tilde{J}^{(n)}(\varphi, t)=\int \frac{\sin ^{n} \varphi \tilde{H}_{j}^{(n+2)}}{\left(\tilde{H}_{j}^{(3)}\right)^{n+1}} d \varphi
$$

Functions $\tilde{I}(\varphi, t)$ and $\tilde{J}^{(n)}(\varphi, t)$ are given in Appendix.

To illustrate graphically the formula (4.2) let us take the values $n=2$ and $n=4$; introducing the notations

$$
\begin{aligned}
& \tilde{H}_{j}^{(3)}=\left\{\begin{array}{lr}
\tilde{h}^{3}\left(1+\frac{1}{3} \frac{c^{* 2}}{\tilde{h}^{2}}\right) & \text { for } \quad j=l \\
\tilde{h}^{3}\left(1-\frac{2}{3} \frac{c^{* 2}}{\tilde{h}^{2}}\right) & \text { for } \quad j=c
\end{array}\right. \\
& \tilde{H}_{j}^{(4)}= \begin{cases}\tilde{h}^{4}\left(1+\frac{2}{3} \frac{c^{* 2}}{\tilde{h}^{2}}\right) & \text { for } \quad j=l \\
\tilde{h}^{4}\left(1-\frac{10}{9} \frac{c^{* 2}}{\tilde{h}^{2}}\right) & \text { for } \quad j=c\end{cases} \\
& \tilde{H}_{j}^{(6)}= \begin{cases}\tilde{h}^{6}\left(1+\frac{5}{3} \frac{c^{* 2}}{\tilde{h}^{2}}\right) & \text { for } \quad j=l \\
\tilde{h}^{6}\left(1-\frac{7}{3} \frac{c^{* 2}}{\tilde{h}^{2}}\right) & \text { for } \quad j=c\end{cases}
\end{aligned}
$$


we will present in Figs 3-6 the graphs of the pressure distributions.

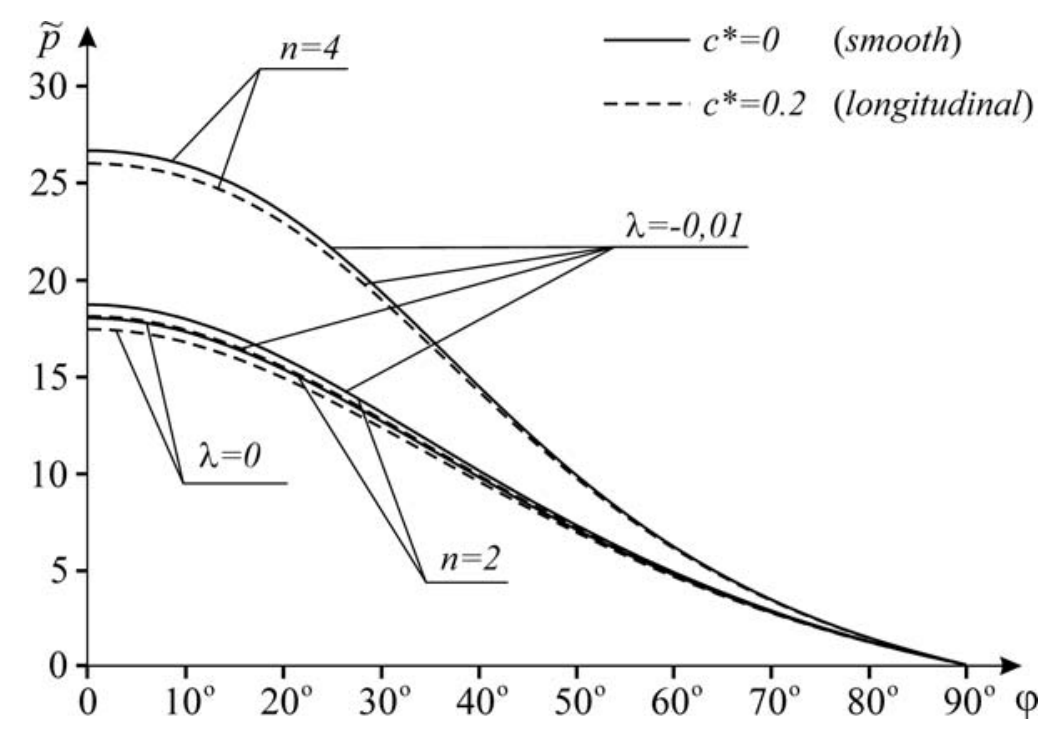

Fig.3. Dimensionless pressure distribution in the spherical bearing between rough surfaces for longitudinal roughness for $\lambda=-0.01$ and $\lambda=0$ and $\varepsilon=0.5$.

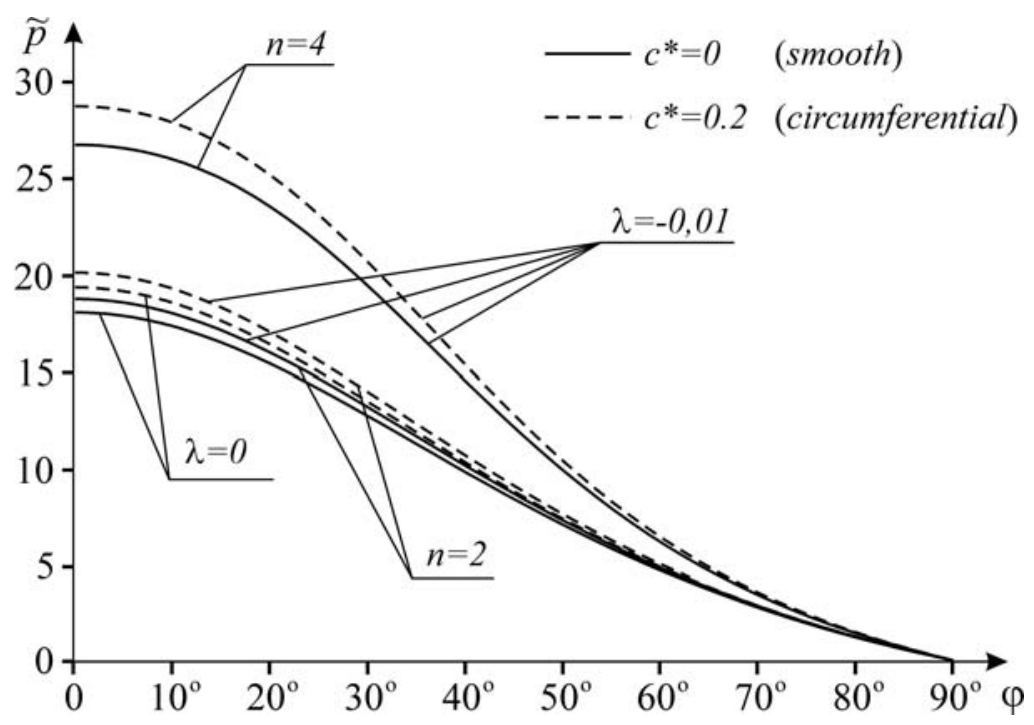

Fig.4. Dimensionless pressure distribution in the spherical bearing between rough surfaces for circumferential roughness for $\lambda=-0.01$ and $\lambda=0$ and $\varepsilon=0.5$. 


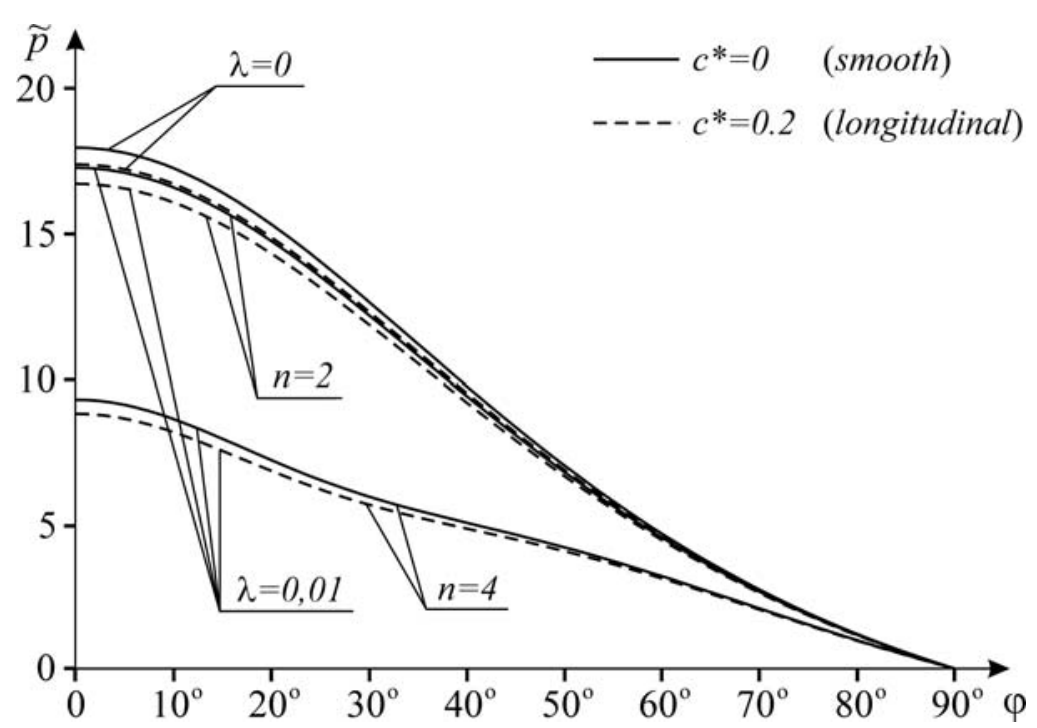

Fig.5. Dimensionless pressure distribution in the spherical bearing between rough surfaces for longitudinal roughness for $\lambda=0$ and $\lambda=0.01$ and $\varepsilon=0.5$.

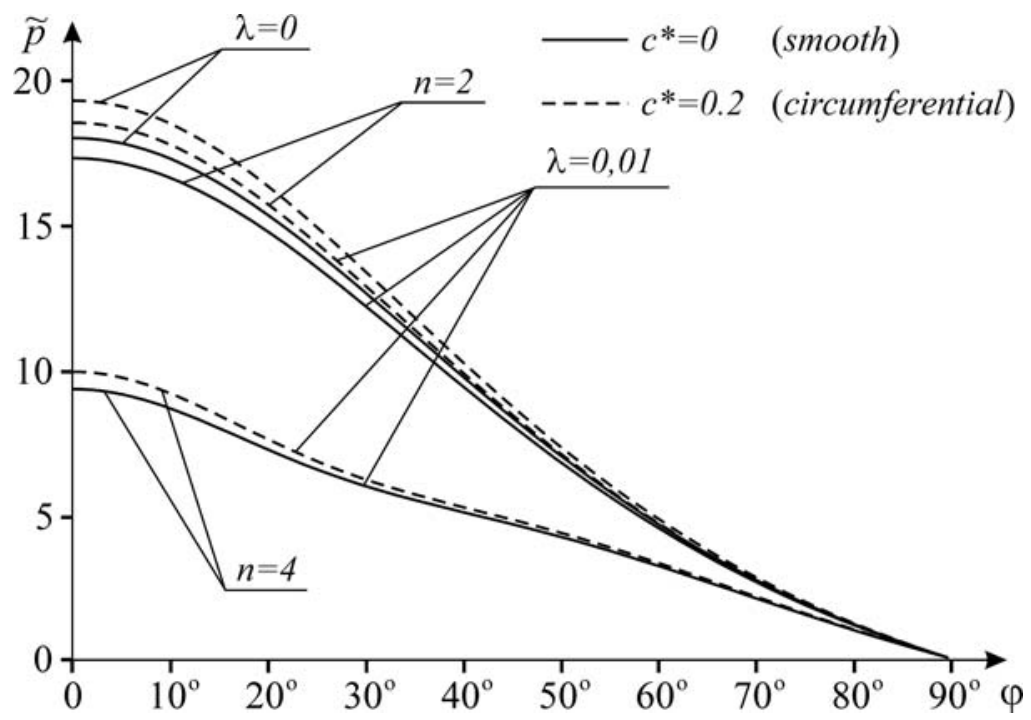

Fig.6. Dimensionless pressure distribution in the spherical bearing between rough surfaces for circumferential roughness for $\lambda=0$ and $\lambda=0.01$ and $\varepsilon=0.5$.

\section{Conclusions}

In this paper the Ellis type of pseudo-plastic lubricants flowing in a clearance of the thrust curvilinear bearing with rough surfaces was analysed. After general considerations the modified Reynolds equations are derived. The general solutions for these equations are searched in a form of the sum of two components; the first of them represents the Newtonian approximation, whereas the other represents the nonNewtonian correction. As a result the general formula for pressure distribution in the spherical bearing between rough surfaces is obtained. The obtained results are relatively simple and may be used in practical applications. 
From calculations and their graphic presentations it that follows the pressure distributions are dependent on the rheological parameters $\tau_{1 / 2}$ and $n$ or $\lambda$ and $n$. For the squeeze film spherical bearing the pressures increase with a decrease of the $\lambda$ values with respect to the suitable values of Newtonian lubricants $(\lambda=0)$. The pressure increases with an increase of $n$ for negative values of $\lambda$ and decreases for positive values of $\lambda$. The bearing surface roughness, expressed by $c^{*}$, results in some small increase of the pressure distribution values for circumferential roughness and in a small decrease for longitudinal roughness.

\section{Nomenclature}

$$
\begin{aligned}
& \boldsymbol{A}_{I} \text { - the first Rivlin-Ericksen kinematic tensor } \\
& c \text { - maximum asperity deviation } \\
& c^{*} \text { - nondimensional roughness parameter } \\
& E(\bullet) \text { - expectancy operator } \\
& e(t) \text { - bearing eccentricity or bearing squeezing } \\
& f\left(h_{s}\right) \text { - probability density distribution function } \\
& H \text { - film thickness } \\
& h(x, t) \quad \text { - nominal film thickness } \\
& h_{s}(x, \vartheta, \xi) \text { - random deviation of film thickness } \\
& p \text { - pressure } \\
& R, R(x) \quad \text { - local radius of the lower bearing surface } \\
& r \text { - radius } \\
& v_{x}, v_{y} \text { - velocity components } \\
& x, y \text { - orthogonal coordinate } \\
& \varepsilon \text { - squeezing ratio } \\
& \vartheta \text { - angular coordinate } \\
& \mu-\text { coefficient of viscosity } \\
& \xi \text { - random variable } \\
& \rho-\text { fluid density }
\end{aligned}
$$

\section{Appendix}

The auxiliary formulae for Newtonian fluid and smooth surfaces

$$
\tilde{I}_{N}(\varphi, t)=-\frac{1}{2 u^{2} \varepsilon}, \quad \tilde{J}_{N}(\varphi, t)=\frac{1}{\varepsilon}\left(-\frac{1}{4 u^{4}}+\frac{1}{8 u^{4} \varepsilon^{2}}-\frac{1}{3 u^{3} \varepsilon^{2}}+\frac{1}{4 u^{4} \varepsilon^{2}}\right) .
$$

The auxiliary coefficients for the roughness surfaces are given

$$
q_{j}^{(3)}=\left\{\begin{array}{ccc}
\frac{1}{3} c^{* 2} & \text { for } & j=l, \\
-\frac{2}{3} c^{* 2} & \text { for } & j=c,
\end{array}\right.
$$




$$
\begin{gathered}
q_{j}^{(4)}=\left\{\begin{array}{lll}
\frac{2}{3} c^{* 2} & \text { for } & j=l, \\
-\frac{10}{9} c^{* 2} & \text { for } & j=c,
\end{array}\right. \\
q_{j}^{(6)}=\left\{\begin{array}{lll}
\frac{5}{3} c^{* 2} & \text { for } & j=l, \\
-\frac{7}{3} c^{* 2} & \text { for } & j=c .
\end{array}\right.
\end{gathered}
$$

The auxiliary formulae for the Ellis fluid $(n=2)$ :

- for longitudinal roughness

$$
\begin{aligned}
& \tilde{I}_{l}(\varphi, t)=\frac{1}{\varepsilon}\left(\frac{\ln u}{q_{l}^{(3)}}-\frac{\ln \left(u^{2}+q_{l}^{(3)}\right)}{2 q_{l}^{(3)}}\right), \\
& \tilde{J}_{l}^{(2)}(\varphi, t)=-\frac{1}{8 \varepsilon^{3}\left(q_{l}^{(3)}\right)^{3}}\left\{\frac{\left(q_{l}^{(3)}\right)^{2}\left(2 u+2 \varepsilon^{2}+q_{l}^{(3)}-1\right)\left(q_{l}^{(3)}-q_{l}^{(4)}\right)}{\left(u^{2}+q_{l}^{(3)}\right)^{2}}+\right. \\
& +2\left(2 \varepsilon^{2}-1\right) \ln \left(u^{2}+q_{l}^{(3)}\right) q_{l}^{(4)}-\arctan \frac{u}{\sqrt{q_{l}^{(3)}}} \sqrt{q_{l}^{(3)}}\left(q_{l}^{(3)}+3 q_{l}^{(4)}\right)+ \\
& \left.+4\left(1-2 \varepsilon^{2}\right) \ln u q_{l}^{(4)}-\frac{q_{l}^{(3)}\left[u q_{l}^{(3)}+2\left(q_{l}^{(3)}\right)^{2}+\left(3 u+4 \varepsilon^{2}-2\right) q_{l}^{(4)}\right]}{u^{2}+q_{l}^{(3)}}\right\},
\end{aligned}
$$

- for circumferential roughness

$$
\begin{aligned}
& \tilde{I}_{c}(\varphi, t)=\frac{1}{\varepsilon}\left(\frac{\ln u}{q_{c}^{(3)}}-\frac{\ln \left(u^{2}+q_{c}^{(3)}\right)}{2 q_{c}^{(3)}}\right), \\
& \tilde{J}_{c}^{(2)}(\varphi, t)=-\frac{1}{8 b^{3} \varepsilon^{3}}\left\{-\sqrt{b} \arctan \frac{u}{\sqrt{b}}\left(b-3 q_{c}^{(4)}\right)+4\left(1-2 \varepsilon^{2}\right) \ln u q_{c}^{(4)}+2\left(1-2 \varepsilon^{2}\right) \ln \left(u^{2}-b\right) q_{c}^{(4)}+\right. \\
& \left.+\frac{b^{2}\left(2 u-b+2 \varepsilon^{2}-1\right)\left(b+q_{c}^{(4)}\right)}{\left(u^{2}-b\right)^{2}}-\frac{b\left[(u-2 b) b+\left(2-3 u-4 \varepsilon^{2}\right) q_{c}^{(4)}\right]}{u^{2}-b}\right\} .
\end{aligned}
$$

The auxiliary formulae for the Ellis fluid $(n=4)$ : 
- for longitudinal roughness

$$
\begin{aligned}
& \tilde{I}_{l}(\varphi, t)=\frac{1}{\varepsilon}\left(\frac{\ln u}{q_{l}^{(3)}}-\frac{\ln \left(u^{2}+q_{l}^{(3)}\right)}{2 q_{l}^{(3)}}\right), \\
& \tilde{J}_{l}^{(4)}(\varphi, t)=-\frac{1}{384 \varepsilon^{5}\left(q_{l}^{(3)}\right)^{5}}\left\{\frac { 2 4 ( q _ { l } ^ { ( 3 ) } ) ^ { 4 } ( q _ { l } ^ { ( 3 ) } - q _ { l } ^ { ( 6 ) } ) } { ( u ^ { 2 } + q _ { l } ^ { ( 3 ) } ) ^ { 4 } } \left[3 \varepsilon^{2}+2 \varepsilon^{4}-1+\right.\right. \\
& \left.+u\left(6 \varepsilon^{2}-4\right)+\left(4 u+3 \varepsilon^{2}-6\right) q_{l}^{(3)}+\left(q_{l}^{(3)}\right)^{2}\right]-192\left(1-3 \varepsilon^{2}+2 \varepsilon^{4}\right) \ln q_{l}^{(6)}+ \\
& +96\left(1-3 \varepsilon^{2}+2 \varepsilon^{4}\right) \ln \left(u^{2}+q_{l}^{(3)}\right) q_{l}^{(6)}+3 \sqrt{q_{l}^{(3)}} \arctan \frac{u}{\sqrt{q_{l}^{(3)}}}\left[35\left(2-3 \varepsilon^{2}\right) q_{l}^{(6)}+\right. \\
& \left.+6\left(q_{l}^{(3)}\right)^{2}-5 q_{l}^{(3)}\left(3 \varepsilon^{2}-2 q_{l}^{(6)}-2\right)+\right]+\frac{3 q_{l}^{(3)}}{u^{2}+q_{l}^{(3)}}\left[6 u\left(q_{l}^{(3)}\right)^{2}-5 u q_{l}^{(3)}\left(3 \varepsilon^{2}-2 q_{l}^{(6)}-2\right)+\right. \\
& \left.+\left(70 u+96 \varepsilon^{2}-105 u \varepsilon^{2}-64 \varepsilon^{4}-32\right) q_{l}^{(6)}\right]+\frac{2\left(q_{l}^{(3)}\right)^{2}}{\left(u^{2}+q_{l}^{(3)}\right)^{2}}\left[6 u\left(q_{l}^{(3)}\right)^{2}+\right. \\
& \left.+24\left(q_{l}^{(3)}\right)^{3}-5 u q_{l}^{(3)}\left(3 \varepsilon^{2}-2 q_{l}^{(6)}-2\right)+q_{l}^{(6)}\left(70 u+72 \varepsilon^{2}-105 u \varepsilon^{2}-48 \varepsilon^{4}-24\right)\right]+ \\
& \left.\left.+q_{l}^{(6)}\left(4-12 \varepsilon^{2}+8 \varepsilon^{4}+7 u\left(3 \varepsilon^{2}-2\right)\right)\right]\right\}, \\
& +\frac{\left(q_{l}^{(3)}\right)^{3}}{\left(u^{2}+q_{l}^{(3)}\right)^{3}}\left[8\left(q_{l}^{(3)}\right)^{3}+u q_{l}^{(3)}\left(3 \varepsilon^{2}-2 q_{l}^{(6)}-2\right)+2\left(q_{l}^{(3)}\right)^{2}\left(9 u+6 \varepsilon^{2}-2 q_{l}^{(6)}-12\right)+\right.
\end{aligned}
$$

- for circumferential roughness

$$
\tilde{I}_{c}(\varphi, t)=\frac{1}{\varepsilon}\left(\frac{\ln u}{q_{c}^{(3)}}-\frac{\ln \left(u^{2}+q_{c}^{(3)}\right)}{2 q_{c}^{(3)}}\right),
$$




$$
\begin{aligned}
& \tilde{J}_{c}^{(4)}=\frac{1}{384 b^{5} \varepsilon^{5}}\left\{192 q_{c}^{(6)}\left(3 \varepsilon^{2}-2 \varepsilon^{4}-1\right) \ln u+96 q_{c}^{(6)}\left(1-3 \varepsilon^{2}+2 \varepsilon^{4}\right) \ln \left(u^{2}-b\right)+\right. \\
& -\frac{24 b^{4}\left(b+q_{c}^{(6)}\right)}{\left(u^{2}-b\right)^{4}}\left[1+b^{2}-3 \varepsilon^{2}+2 \varepsilon^{4}-3 b\left(\varepsilon^{2}-2\right)+u\left(6 \varepsilon^{2}-4 b-4\right)\right]+ \\
& +3 \sqrt{b} \operatorname{arctanh} \frac{u}{\sqrt{b}}\left[b\left(15 \varepsilon^{2}+6 b-10\right)-5 q_{c}^{(6)}\left(21 \varepsilon^{2}+2 b-14\right)\right]+\frac{8 b^{4}}{\left(u^{2}-b\right)^{3}}\left(2 u b-24 b^{2}+\right. \\
& +18 u b^{2}-8 b^{3}-3 u b \varepsilon^{2}+12 b^{2} \varepsilon^{2}+4 q_{c}^{(6)}-14 u q_{c}^{(6)}+2 u b q_{c}^{(6)}-4 b^{2} q_{c}^{(6)}-12 \varepsilon^{2} q_{c}^{(6)}+ \\
& \left.+21 u \varepsilon^{2} q_{c}^{(6)}+8 \varepsilon^{4} q_{c}^{(6)}\right)-\frac{2 b^{2}}{\left(u^{2}-b\right)^{2}}\left[10 u b-6 u b^{2}+24 b^{3}-15 u b \varepsilon^{2}+q_{c}^{(6)}(24-70 u+10 u b+\right. \\
& \left.\left.-72 \varepsilon^{2}+105 u \varepsilon^{2}+48 \varepsilon^{4}\right)\right]-\frac{3 b}{u^{2}-b}\left[18 u b^{3}-30 u b^{2}+45 u b^{2} \varepsilon^{2}+q_{c}^{(6)}(210 u b-96 b+\right. \\
& \left.\left.\left.-30 u b^{2}+288 b \varepsilon^{2}-315 u b \varepsilon^{2}-192 b \varepsilon^{4}\right)\right]\right\} .
\end{aligned}
$$

\section{References}

[1] Covey G.H. and Stanmore B.R. (1981): Use of the parallel-plate plastometer for the characterisation of viscous fluids with a yield stress. - J. Non-Newton. Fluid Mech. vol.8, pp.249-260.

[2] Dai G. and Bird R.B. (1981): Radial flow of Bingham fluid between two fixed circular disks. - J. Non-Newton. Fluid Mech. vol.8, pp.349-355.

[3] Lipscomb C.C. and Denn M.M. (1984): Flow of Bingham fluids in complex geometries. - J. Non-Newt. Fluid Mech., vol.14, No.3, pp.337-349.

[4] Walicki E. and Walicka A. (1998): Mathematical modelling of some biological bearings. - Smart Materials and Structures, Proc. 4th European and $2^{\text {nd }}$ MiMR Conference, Harrogate, UK, 6-8 July 1998, pp.519-525.

[5] Dorier C. and Tichy J. (1992): Behaviour of a Bingham-like viscous fluid in lubrication flows. - J. Non-Newt. Fluid Mech., vol.45, No.3, pp.291-350.

[6] Wada S. and Hayashi H. (1971): Hydrodynamic lubrication of journal bearings by pseudo-plastic lubricants, (Pt 1, Theoretical studies). - Bull. JSME, vol.14, No.69, pp.268-278.

[7] Wada S. and Hayashi H. (1971): Hydrodynamic lubrication of journal bearings by pseudo-plastic lubricants. (Pt 2 , Experimental studies). - Bull. JSME, vol.14, No.69, pp.279-286.

[8] Swamy S.T.N., Prabhu B.S. and Rao B.V.A. (1975): Stiffness and damping characteristics of finite width journal bearing with a non-Newtonian film and their application to instability prediction. - Wear, vol.32, pp.379-390.

[9] Rajalingham C., Rao B.V.A. and Prabu S. (1978): The effect of a non-Newtonian lubricant on piston ring lubrication. - Wear, vol.50, pp.47-57.

[10] Walicka A. (2002): Rotational Flows of Rheologically Complex Fluids in Thin Channels (in Russian). - Zielona Gora: University Press.

[11] Walicki E. (2005): Rheodynamics of Slide Bearings Lubrication (in Polish). - Zielona Gora: University Press.

[12] Walicka A. (1994): Micropolar Flow in a Slot Between Rotating Surfaces of Revolution. - Zielona Góra: TU Press.

[13] Walicki E. and Walicka A. (1998): Mathematical modelling of some biological bearings. - Smart Materials and Structures, Proc. 4th European and $2^{\text {nd }}$ MiMR Conference, Harrogate, UK, 6-8 July 1998, pp.519-525. 
[14] Khonsari M.M. and Dai F. (1992): On the mixture flow problem in lubrication of hydrodynamic bearing: small solid volume fraction. - STLE Trib. Trans., vol.35, No.1, pp.45-52.

[15] Agrawal V.K. (1970): Effect of lubricant inertia on squeeze film in spherical bearing. - Jap. J. Appl. Phys., vol.9, No.7, pp.831-833.

[16] Gould F. (1975): High-pressure spherical squeeze films. - J. Lubric. Technol. Trans. ASME, ser. F, vol.97, No.1, pp.207208.

[17] Murti P.R.K. (1975): Squeeze film in curved circular plates. - J. Lubric. Technol. Trans. AME, ser F, vol.97, No.4, pp.650-654.

[18] Vora K.H. (1980): Behaviour of squeeze film between curved circular plates with a concentric circular pocket. Wear, vol.65, pp.35-38.

[19] Walicka A. (1989): Accurate and Asymptotic Solution of Simplified Sets of Equations Describing the Motion of Viscous Fluids in a Slot Bounded by Two Co-axial Surfaces of Revolution (in Polish). - Warszawa: PWN.

[20] Christensen H. (1970): Stochastic model for hydrodynamic lubrication of rough surfaces. - Proc. Inst. Mech. Engrs, vol.184, pt 1, pp.1013-1022.

[21] Bujurke N.M., Kudenatti R.B. and Awati V.B. (2007): Effect of surface roughness on squeeze film poroelastic bearings with special reference to synovial joints. - Mathematical Biosciences, vol.209, pp.76-89.

[22] Lin J.-R. (2000): Surfaces roughness effect on the dynamic stiffness and damping characteristics of compensated hydrostatic thrust bearings. - Int. J. Machine Tools Manufact., vol.40, pp.1671-1689,.

[23] Lin J.-R. (2001): The effect of couple stresses in the squeeze film behavior between isotropic rough rectangular plates. - Int. J. Appl. Mech. Eng., vol.6, No.4, pp.1007-1024.

[24] Prakash J. and Tiwari K. (1984): An analysis of the squeeze film between rough porous rectangular plates with arbitrary porous wall thickness. - Journal of Tribology, Trans. ASME, vol.106, No.2, pp.218-222.

[25] Prakash J. and Tiwari K. (1985): Effects of surface roughness on the squeeze film between rectangular porous annular disc with arbitrary porous wall thickness. - Int. J. Mech. Sci., vol.27, No.3, pp.135-144.

[26] Walicka A. (2009): Surface roughness effects in a curvilinear squeeze film bearing lubricated by a power-law fluid. - Int. J. Appl. Mech. Engng, vol.14, No.1, pp.277-293.

[27] Walicka A. (2012): Porous curvilinear squeeze film bearing with rough surfaces lubricated by a power-law fluid.Journal of Porous Media, vol.15, No.1, pp.29-49.

[28] Walicka A. and Walicki E. (2002): Surface roughness effect on the pressure distribution in curvilinear thrust bearings. - Exploitation Problems of Machines, vol.131, No.3, pp.157-167.

[29] Walicka A. and Walicki E. (2002): Couple stress and surface roughness effects in curvilinear thrust bearings. Int. J. Appl. Mech. Engng, vol.7, Spec. Issue: SITC, pp.109-117.

[30] Gururajan K. and Prakash J. (1999): Surface roughness effects in infinitely long porous journal bearing. - Journal of Tribology, Trans. ASME, vol.121, No.1, pp.139-147.

[31] Ellis S.B. (1927): Thesis, Lafayette College, Pa. Citted in: Matsuhisa S., Bird R.B. (1965): Analytical and numerical solutions for laminar flow of the Non-Newtonian Elis fluid. - AiChE Journal, pp.588-595.

[32] Rotem Z. and Shinnar R. (1961): Non-Newtonian flow between parallel boundaries in linear movements. - Chem. Eng. Sie., vol.15, pp.130-143.

[33] Walicka A. (2002a): Rheodynamics of Non-Newtonian Fluids Flow in Straight and Curved Channels (in Polish), Zielona Gora: University Press.

[34] Walicki E. (1977): Viscous Fluid Flow in Slots of Thrust Bearings. - Bydgoszcz: AT-R. Press.

[35] Christensen H. and Tønder K. (1971): The hydrodynamic lubrication of rough bearing surfaces of finite width. ASME, J. Lubric. Technol., vol.93, No.2, pp.324-330.

[36] Christensen H. and Tønder K. (1973): The hydrodynamic lubrication of rough journal bearings. - ASME, J. Lubric. Technol., vol.95, No.1, pp.166-172. 
[37] Whorlow R.W. (1992): Rheological Techniques (Sec. Edition). - Ellis Horwood, New York.

[38] Walicka, A., Walicki, E. and Ratajczak, M. (1999): Pressure distribution in a curvilinear thrust bearing with pseudo-plastic lubricant. - Appl. Mech. Enging. 4 (sp. Issue), pp.81-88.

[39] Walicka, A., Walicki, E. and Ratajczak, M. (2000): Rotational inertia effects in a pseudo-plastic fluid flow between non-coaxial surfaces of revolution. - Proc. 4th Minsk Int. Heat Mass Transfer Forum (May 22-27, 2000 Minsk Belarus), pp.19-29.

Received: June 12, 2016

Revised: July 2, 2016 